\title{
Dissociating restart cost and mixing cost in task switching
}

\author{
Edita Poljac $\cdot$ Iring Koch $\cdot$ Harold Bekkering
}

Received: 12 September 2007 / Accepted: 10 April 2008 / Published online: 30 April 2008

(C) The Author(s) 2008

\begin{abstract}
Three experiments investigated the cognitive mechanisms underlying the restart cost and mixing cost in task switching. To this aim, the predictability of task order was varied (unpredictable in Experiment 1 and predictable in Experiments 2 and 3) across experiments, which employed a multiple-trial paradigm. Verbal cues for color and shape matching tasks were presented before a run of four trials. Focusing on task-repetition runs only, we measured restart cost as the difference in performance between trials 1 and 2 and mixing cost as the difference in performance on the non-cued trials under mixed-tasks conditions (Experiments 1 and 2) and single-task conditions (Experiment 3). The restart cost was observed under mixed-tasks conditions with both unpredictable and predictable task orders but not under the single-task condition. In contrast, the mixing cost was observed under the mixed-tasks condition with unpredictable task order only (Experiment 1). This finding implies that the optimal task execution on repetition trials depends on how predictable the identity of the approaching task is. Therefore, we suggest that mixing cost arises from limited preparation on repetition trials when task order is unpredictable, while restart cost arises from processes involved in cue-based task activation that is needed to resolve task interference. Together, these data
\end{abstract}

E. Poljac $\cdot$ H. Bekkering

Radboud University Nijmegen, Nijmegen, The Netherlands

I. Koch

RWTH Aachen University, Aachen, Germany

E. Poljac $(\bowtie)$

Nijmegen Institute for Cognition and Information, P.O. Box 9104, 6500 HE Nijmegen, The Netherlands e-mail: e.poljac@nici.ru.nl suggest that restart cost and mixing cost are based on dissociable mechanisms.

\section{Introduction}

Cognitive flexibility allows for context-specific and goaldirected action. This cognitive flexibility can be studied using task-switching paradigms, which usually involve switching between two tasks according to different, arbitrary stimulus-response (S-R) mappings (e.g., Monsell, 2003, for a review). Mostly, the stimuli used in task-switching paradigms are bivalent, that is, consisting of two dimensions (e.g., color and shape), each relevant to one of the tasks. This stimulus bivalency has two important consequences. First, since bivalent stimuli do not specify which of the two tasks is expected to be executed, predictable task orders or, most often, cues are used to specify tasks. Second, both the relevant and the irrelevant stimulus features are visually present, so that the currently irrelevant stimulus feature can interfere with the execution of the required task (e.g., Allport, Styles, \& Hsieh, 1994). The task execution under these conditions is often accompanied by performance costs. The present study aimed at examining two performance costs that are both related to task repetitions, restart cost and mixing cost, using bivalent stimuli in cued task switching.

The phenomenon of restart cost refers to the response delay on cued trials compared to trials that were not preceded by cues (e.g., Allport \& Wylie, 2000; Altmann \& Gray, 2002; Gopher, Armony, \& Greenshpan, 2000; Poljac, De Haan, \& Van Galen, 2006). Generally, in these studies, the execution of a run of trials belonging to one of the two tasks is interrupted by a cue that informs the participant which task to perform next. This can imply a task switch 
(when the cue changes the task) or a task repetition (when the cue does not change the task). Both task-switch and task-repetition runs showed significant performance costs. The cost associated with a task repetition is called restart cost. ${ }^{1}$ Research so far has shown that restart cost can be reduced with increased preparation time in task repetitions, the so-called preparation effect, even though restart cost is usually not eliminated altogether (e.g., Altmann, 2004; Poljac et al., 2006).

A theoretical account for restart cost was recently proposed by Altmann (2002). He suggested that interference between two competing tasks calls for cognitive adaptations that enable the system to switch between the competing tasks. According to Altmann, these cognitive adaptations are reflected in a decay of task-set activation when executing runs of trials belonging to the same task, observed as a gradual increase in reaction times (RTs) across the successive trials (within-run slowing), and in the recovery of the activation through cue encoding processes. During the encoding of the appropriate cue, the activation of the relevant task representation rises until it is higher than the activation of the previous, now superseded representation. The effort that it takes to (re)build the episodic representation in memory is reflected in restart cost (and switch cost, see footnote 1). Accordingly, ample preparation time given to (re)build the representation would result in the performance improvement on cued trials reflected in preparation effects.

A phenomenon that also considers task repetition performance and, therefore, relates to restart cost is mixing cost. Many studies reported mixing cost observed as slower responses on repetition trials under mixed-tasks conditions than under single-task conditions (e.g., Los, 1996; Rubin \& Meiran, 2005). Los proposed that this mixing cost arises due to mental load that is higher under the mixed conditions, where two tasks alternate, than under the single-task conditions (see also Rogers \& Monsell, 1995). Recently, however, Rubin and Meiran found no evidence for sustained working memory demands contributing to mixing cost. The findings of their study implied the transient competition between tasks as the main contributor to mixing cost (see also Braver, Reynolds, \& Donaldson, 2003). Consistent with this implication, Koch, Prinz, and Allport (2005) demonstrated that increasing the interference between tasks on the level of varied stimulus-task associations contributed to mixing cost. Accordingly, it seems that mixing cost relates to the interference arising from bivalent stimuli affording both tasks, so that unintentional activation

\footnotetext{
${ }^{1}$ The difference in performance on cued trials involving a task switch and those involving a task repetition is the so-called switch cost. Here we focus on restart cost and mixing cost (for details on definitions of switch cost see Altmann, 2007).
}

of the competing task due to stimulus bivalency can increase the interference between the tasks involved (e.g., Allport \& Wylie, 2000; Koch \& Allport, 2006; Rubin \& Koch, 2006; Waszak, Hommel, \& Allport, 2003).

Altogether, it seems that both restart and mixing cost relate in some way to the interference existing between the competing tasks in task switching. The present study investigated in more detail the nature of the mechanisms behind these two performance costs observed for task repetitions. For this purpose, we varied interference between the tasks by manipulating task predictability, which referred to the extent to which the participant could identify the upcoming task before the stimulus presentation on cued trials.

We used a multiple-trials paradigm (e.g., Altmann \& Gray, 2002), in which color matching (task A) or shape matching (task B) was required. In this paradigm, runs of trials including one of the two tasks are interrupted by cue presentations, which indicate a task switch or a task repetition. We assumed here that cue-based task activation, a cognitive adaptation to task interference, is reflected in restart cost. Our reasoning was that while on the first trials, both a task cue and a stimulus need to be processed before giving a correct response, only stimulus processing is required for adequate task execution on the following trials in the run. Accordingly, the possible difference in performance between first trials and following trials after a task repetition should reflect processes related to cue-based task activation. In addition, differently from most other studies so far, the present study addressed in more detail the dynamics of performance in cued task-repetition trials associated with successive execution of the same task that allows the cognitive system to improve the repetition performance (e.g., Meiran, Chorev, \& Sapir 2000).

Furthermore, we assumed that mixing cost reflects task interference. Mixing cost was measured by comparing the performance in task-repetition runs under the mixed-tasks (Experiments 1 and 2) and single-task (Experiment 3) conditions. The presentation of task runs, which we will call task order hereafter, was varied as a function of task predictability (see Table 1). While unpredictable tasks were specified with cues in Experiment 1, Experiment 2 additionally included a predictable task order, and Experiment 3 induced even a complete separation between the two tasks. We assumed that the identity of the approaching task was less predictable in Experiment 1 than in both Experiment 2 and Experiment 3.

We expected that less predictable identity of the approaching task would require stronger cue-based task activation, which would be reflected in higher restart cost in Experiment 1 than in both Experiments 2 and 3. We also expected that task interference should be reflected in mixing cost, which we expected to observe in task-repetition trials 
Table 1 Overview of task conditions and task order in Experiments $1-3$

\begin{tabular}{lll}
\hline Experiment & Task condition & Task order \\
\hline 1 & Mixed & Unpredictable \\
2 & Mixed & Predictable \\
3 & Single & Predictable \\
\hline
\end{tabular}

under the mixed-tasks conditions (Experiments 1 and 2) relative to the single-task baseline condition (Experiment 3).

\section{Experiment 1}

This experiment examined cue-based task activation in situations of task ambiguity induced by stimulus bivalency and unpredictable task order. This was done by focusing on restart cost, measured as the difference in performance between trials 1 and 2 in task-repetition runs. More specifically, we examined the dynamics of restart cost. Unlike previous studies, in the present study a task could be repeatedly restarted one to three times. These successive task-restarts implied continuous execution of one of the two tasks for a certain number of trials. In this way, we explored the issue whether continued execution of the same task (even with cue presentations in between) allows the cognitive system to reduce the cost associated with the restart of this task (restart cost). If a relatively long execution of a task (with a relatively larger distance from a previous task switch) allows the cognitive system to reduce the restart cost, then this should be observed as a decrease in restart cost with an increasing number of task-repetition runs.

\section{Method}

\section{Participants}

Sixty participants, 40 women and 20 men, were paid for taking part in this experiment. Their ages ranged between 18 and 42 years, with a mean age of 22.3 years. Nine participants were left-handed, and the remaining 51 were righthanded. All participants had normal or corrected-to-normal vision. Eight additional participants were excluded because their accuracy was below $90 \%$.

\section{Stimuli and tasks}

The stimuli were selected from four different geometric figures (a square, a triangle, a circle, and a hexagon) and were displayed in one of four different colors (red, blue, yellow, or green). On each trial, a reference figure and four match figures were presented. The reference figure was displayed in the upper half of the screen, while the four match figures were displayed simultaneously in the lower half of the screen. The color-shape combination of the stimuli was randomly chosen for both tasks with two restrictions. First, no simultaneous occurrence of the same shape or color was allowed among the four match figures within a trial. Second, the exact match (in both shape and color) was not allowed between the reference and match figures (i.e., stimuli were always "incongruent"). The tasks were to match either the color or the shape of the reference figure to one of the four match figures. Written cues, printed in uppercase 32-point Times New Roman font, were used to announce the upcoming task. The cue was either the word "KLEUR" or the word "VORM" (the Dutch equivalents for "color" and "shape").

\section{Procedure}

The experiment was run in a single session with one participant at a time. The participant was seated in front of a screen of a Pentium $650 \mathrm{MHz}$ (17 in. effective screen) at a distance of approximately $60 \mathrm{~cm}$. Written instructions were displayed on the screen, and the experiment was also verbally explained. The participant was asked to respond as quickly and accurately as possible by pressing one of the four buttons on a button box with either the index or the middle finger of either the right or left hand. The four buttons corresponded to the location of the four match figures that were presented on the screen. Both the reference and the match figures remained on the screen until the participant gave a response or until a maximum response time of $3 \mathrm{~s}$ had elapsed (no-response). In the latter case, the participant received feedback to respond more quickly.

After reading the instructions, the participant started practicing the tasks. The actual tasks were organized in runs of four trials (see Fig. 1). Either the color or the shape of the reference figure needed to be matched. The task cue appeared at the screen center at the beginning of a task run and disappeared after $300 \mathrm{~ms}$. Immediately after the cue disappeared, the first of the four stimuli in the run was presented until a response (or feedback if too slow) was given. The response-stimulus interval (RSI) was fixed at $100 \mathrm{~ms}$, after which period a new stimulus appeared, followed by a response. This was repeated until the fourth response. After the last response and the $100 \mathrm{~ms}$ of the response-cue interval (RCI), a new run started, again preceded by a task cue. No switching between the two tasks occurred within runs. Participants first completed the 10 practice runs and then a total of 164 experimental runs, divided over four consecutive parts. The first task run of each experimental part was considered as a warming up run. 


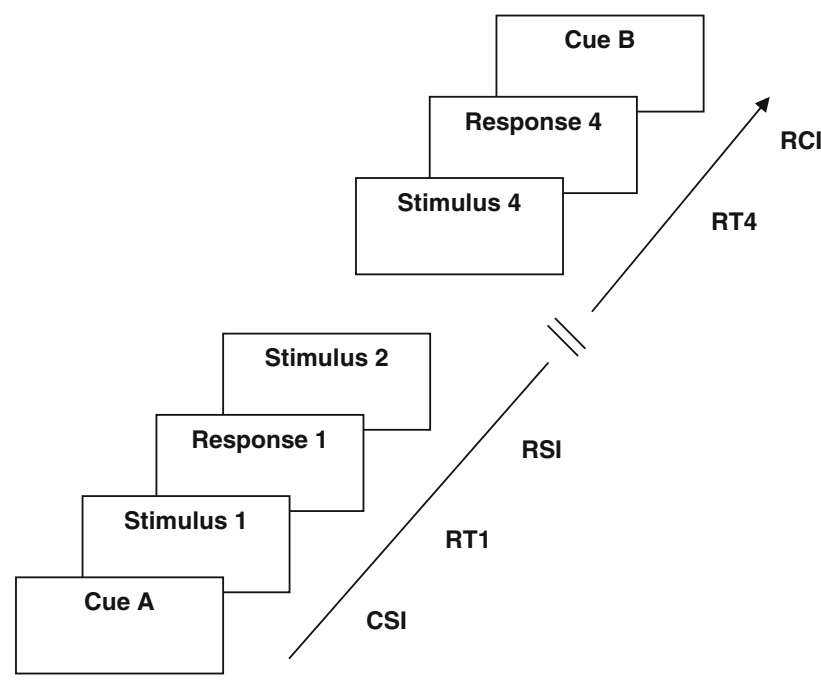

Fig. 1 Schematic description of the event sequence in a task run as used in Experiment 1. A cue for the upcoming task A appears and stays on the screen for $300 \mathrm{~ms}$ of the cue stimulus interval (CSI). Immediately after the CSI, the first stimulus is presented and stays on the screen until a response is given, which results in a recorded reaction time on the first stimulus (RT1). After $100 \mathrm{~ms}$ of the response stimulus interval (RSI), a new stimulus appears, and this sequence is repeated for all four trials of the task run. The last response $(R T 4)$ in the task run is followed by $100 \mathrm{~ms}$ of the response cue interval $(R C I)$, after which period a new task run starts, again preceded by a task cue

\section{Design}

Sixty-four of the experimental runs were so-called switch runs, in which the task differed from the task in the previous run. Next, the 96 repetition runs were 48 first runs, 32 second runs, and 16 third runs. The task in these runs was identical to the task in the preceding run and was repeated for the first, second, or third time, respectively. The two tasks were equally represented in all four types of task runs. The independent variables were Run type (first run, second run, and third run) and Trial (1, 2, 3, and 4). The task-switch runs were excluded from the analysis. ${ }^{2}$ Since the variable Task (color/shape) did not produce any reliable interactions that would change any of our theoretical conclusions, we decided to collapse the data across the Task variable. As dependent variables, RTs were measured for each button

\footnotetext{
${ }^{2}$ Because task switching was not the focus of the present study, we did not include switch runs in the analyses. The switch runs, however, were necessary for introducing task unpredictability. To allow for a comparison of the data between this study and the previous taskswitching studies, the major findings on task switching performance are briefly reported here. A significant switch cost was observed in both Experiment 1 [33 ms; $t(59)=3.52, P<0.005$ ] and Experiment 2 $[144 \mathrm{~ms} ; t(39)=10.04, P<0.001]$. The switch cost was measured by comparing the performance on first trials after a task switch with the performance on first trials after a task repetition (929 vs. $896 \mathrm{~ms}$ and 949 vs. 805 ms, for Experiments 1 and 2, respectively).
}

press, and incorrect responses as well as no-responses were recorded. An alpha level of .05 was used for all statistical tests in this study.

\section{Results}

For RT analysis, we excluded the 10 practice runs, error trials and no-response trials as well as the trials that immediately followed. Furthermore, if within a certain task run all four trials were error trials, then the whole task run that immediately followed was also not included in the analysis. These trial exclusion criteria were applied in all experiments of this study.

The RT data are depicted in Fig. 2. We submitted median RT to a $3 \times 4$ (Run type $\times$ Trial) repeated measures analysis of variance (ANOVA). The ANOVA yielded a significant main effect of trial, $F(3,57)=22.39$, $P<0.001$. The responses on first trials $(896 \mathrm{~ms})$ were slower than the responses on all other trials $(811,814$, and $821 \mathrm{~ms}$ for trials 2,3 , and 4, respectively). A significant RT-difference was observed between first trials and all subsequent trials in the run $[F(1,59)=57.54, P<0.001$; $F(1,59)=65.32, P<0.001 ;$ and $F(1,59)=53.82$, $P<0.001$, for Trial 1 vs. Trial 2, 3, and 4 respectively]. Furthermore, a linear increase in RTs was observed across Trial 2, 3, and 4, which almost reached its significance, $F(1,59)=3.35, P=0.072$. The main effect run type was not significant, $F(2,58)=1.71, P=0.19$.

A significant interaction between run type and trial was observed with $F(6,54)=2.31, P<0.05$. Importantly, Fig. 2 shows that this interaction was due to a linear performance improvement on Trial $1[F \quad(1,59)=8.81$, $P=0.004]$ and no difference in performance on Trial 2, 3, or $4(F<1$ for all) across the first, second, and third task run. A significant restart cost was observed for first $[107 \mathrm{~ms} ; F(1,59)=69.40, P<0.001]$, second $[79 \mathrm{~ms}$; $F(1,59)=31.23, P<0.001]$, and third task run $[68 \mathrm{~ms}$; $F(1,59)=23.86, P<0.001]$.

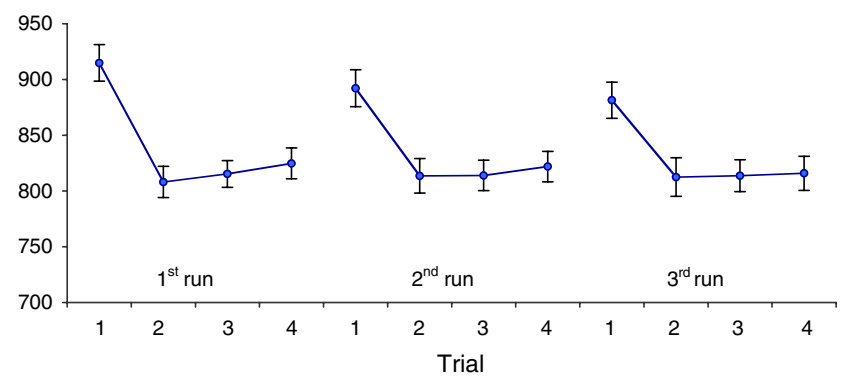

Fig. 2 Means of median response time (ms) and standard error in Experiment 1 as a function of trial number (1/2/3/4) over the three taskrun types (from left to right: first run, second run, and third run) 
Table 2 Error rates (in \%) and standard errors (SE) as a function of trial for the three run types in Experiments 1 and 2

\begin{tabular}{|c|c|c|c|c|c|c|c|c|c|}
\hline \multirow[t]{2}{*}{ Experiment } & \multirow{2}{*}{$\begin{array}{l}\text { Run } \\
\text { type }\end{array}$} & \multicolumn{2}{|c|}{ Trial 1} & \multicolumn{2}{|l|}{ Trial 2} & \multicolumn{2}{|c|}{ Trial 3} & \multicolumn{2}{|l|}{ Trial 4} \\
\hline & & Errors & SE & Errors & SE & Errors & SE & Errors & SE \\
\hline \multirow[t]{3}{*}{ Experiment 1} & 1 st run & 3.33 & 0.63 & 3.40 & 0.42 & 4.03 & 0.44 & 2.95 & 0.39 \\
\hline & 2nd run & 2.19 & 0.39 & 2.71 & 0.37 & 2.03 & 0.35 & 2.71 & 0.41 \\
\hline & 3rd run & 2.40 & 0.45 & 3.23 & 0.72 & 2.19 & 0.64 & 2.92 & 0.55 \\
\hline \multirow[t]{3}{*}{ Experiment 2} & 1st run & 3.18 & 0.56 & 2.97 & 0.53 & 3.07 & 0.44 & 3.13 & 0.54 \\
\hline & 2nd run & 2.08 & 0.40 & 2.81 & 0.48 & 2.34 & 0.45 & 2.03 & 0.43 \\
\hline & 3 rd run & 1.82 & 0.33 & 3.23 & 0.53 & 2.19 & 0.37 & 2.19 & 0.35 \\
\hline
\end{tabular}

In this study, error rates were first transformed using the arcsine transformation (Bishop, Fienberg, \& Holland, 1975) to achieve approximate variance equality. A $3 \times 4$ (Run type $\times$ Trial) repeated measures ANOVA on errors (see Table 2) revealed a significant main effect of run type, $F(2,58)=11.36, P<0.001$. The participants made, on average, more errors in first runs $(3.43 \%)$ than in second $(2.41 \%)$ and third runs $(2.68 \%)$, with $F(1,59)=11.78$, $P=0.001$ and $F(1,59)=20.66, P<0.001$, respectively. The main effect of trial was not significant, $F(3,57)=1.05$, $P=0.38$. In addition, the ANOVA yielded a significant interaction between run type and trial, $\mathrm{F}(6,54)=3.53$, $P<0.01$. While a significant decrease in errors from Trial 3 to Trial 4 was observed in first runs $(F(1,59)=7.89$, $P=0.007)$, an increase in errors from Trial 3 to Trial 4 almost reached significance for both second $[F(1,59)$ $=2.86, P=0.096]$ and third runs $[F(1,59)=3.77$, $P=0.057]$. We have no ready explanation for the somewhat unruly pattern of this particular interaction. The task runs included in the analysis contained on average a low error rate of $2.84 \%$.

\section{Discussion}

The present experiment not only showed restart cost (e.g., Allport \& Wylie, 2000; Altmann \& Gray, 2002; Gopher et al., 2000), but also the persistent nature of this cost. However, as a novel empirical contribution, we observed a decrease in restart cost due to successive repeating of task runs. A similar improvement was also reported in studies using the explicit cueing paradigm, in which cues are presented on each trial. These studies showed that processing on successive repeat trials could improve (e.g., Meiran et al., 2000; Milan, Sanabria, Tornay, \& Gonzalez, 2005, Monsell, Sumner, \& Waters, 2003; Tornay \& Milan, 2001). However, it is not possible to infer from these studies which process profits from task repetitions, since the explicit-cuing paradigm does not distinguish between cuerelated processes and stimulus- and/or response-related processes. The observed improvement on first trials and the corresponding decrease of restart cost implies that it is cuebased task activation that profits from task repetitions (for discussion see Altmann, 2007). In Experiment 2, the role of task predictability in restart cost was investigated.

\section{Experiment 2}

The aim of Experiment 2 was twofold. First, we tested the idea that the restart cost mainly reflects cue-based task activation needed for managing task ambiguity in task switching. In the previous experiment, task ambiguity due to stimulus bivalency was resolved by cue presentations, but the task order was still unpredictable. In the present experiment, however, we introduced predictable task order in similar mixed-tasks conditions. The participants were informed in advance of which task would be required in a certain sequence of four task runs. In this way, although required to switch between task sequences, participants no longer needed to deal with unpredictable task order. If restart cost is mainly generated by cue-based task activation needed to resolve task ambiguity induced by unpredictable task order, then cue presentations in the present experiment should induce significantly smaller restart cost than that in Experiment 1.

As the second aim, we also tested the idea that higher task predictability reduces interference of the competing task, because task predictability can be used for effective task preparation (Koch 2005, 2008). To test this, we compared performance under task-repetition trials between Experiment 2 and Experiment 1, in which the bivalent stimuli occurred in unpredictable task sequences. If task interference arises primarily under situations of task unpredictability, then comparing task repetitions in Experiments 1 and 2 should show mixing cost, reflecting management of the cognitive system with task interference necessary in Experiment 1 but not in Experiment 2.

\section{Method}

\section{Participants}

Forty participants, 32 women and 8 men, were paid for taking part in this experiment. Their ages ranged between 18 and 29 years, with a mean age of 21.3 years. One participant was left-handed, and the remaining 39 were righthanded. All participants had normal or corrected-to-normal vision. Four additional participants were excluded because their accuracy was below $90 \%$.

\section{Stimuli and tasks}

The stimuli and tasks were the same as in Experiment 1. 


\section{Procedure and design}

The procedure and the design of this experiment were almost identical to that of Experiment 1, but making the task order predictable required some procedural and design adaptations. First, the tasks were organized in sequences of four task runs consisting of just one of the two tasks. Such a sequence started with a sequence cue, introducing the task for the upcoming sequence ("color sequence" or "shape sequence"). This cue stayed on the screen as long as a participant needed to prepare for the upcoming task sequence. The participants were explicitly instructed to use this interval to prepare the task sequence as fully as possible. When ready, participants could initiate the sequence of the introduced task, by pressing one of the four buttons. After this button-press and a blank screen for 1,000 ms, the cue for the upcoming task run appeared, just as in Experiment 1 . The structure of task runs was identical to that in Experiment 1, that is, a task cue (for $300 \mathrm{~ms}$ ) followed by four trials. Importantly, a color sequence was always followed by a shape sequence and vice versa. This implies that a sequence always consisted of a task switch run and three subsequent task repetition runs (the first repetition run, the second repetition run, and the third repetition run). Also in this experiment, the analysis included repetition runs only.

In this experiment, participants started practicing by completing four task sequences, after which they completed a total of 56 sequences of the experiment. These experimental sequences were divided over four consecutive parts. The first two task sequences of each part were considered as a warming up sequence. This experimental structure involved an equal number (48) of task runs for task switch, first, second, and third task repetition runs.

\section{Results}

A $3 \times 4$ (Run type $\times$ Trial) repeated measures ANOVA on RTs yielded a significant main effect of trial, $F(3,37)$ $=14.18, P<0.001$. On average, the responses on first trials ( $805 \mathrm{~ms})$ were slower than the responses on second $(759 \mathrm{~ms})$, third $(767 \mathrm{~ms})$, and fourth trial $(778 \mathrm{~ms})$ in the run, with $F(1,39)=31.29, P<0.001 ; F(1,39)=22.73$, $P<0.001$; and $F(1,39)=11.63, P<0.005$, respectively. Furthermore, an increase in RTs was observed across Trial 2,3 , and 4 , with a significant linear trend, $F(1,39)=20.26$, $P<0.001$. Neither the main effect of run type $(F<1)$ nor the interaction $[F(6,34)=1.00, P=0.69]$ was significant.

Importantly, by comparing the performance between Trial 1 and Trial 2 (see Fig. 3), a significant restart cost was observed for first $[54 \mathrm{~ms} ; F(1,39)=18.28, P<0.001]$, second $[47 \mathrm{~ms} ; F(1,39)=27.48, P<0.001]$, and third run $[39 \mathrm{~ms} ; F(1,39)=17.70, P<0.001]$, but, unlike Experi-

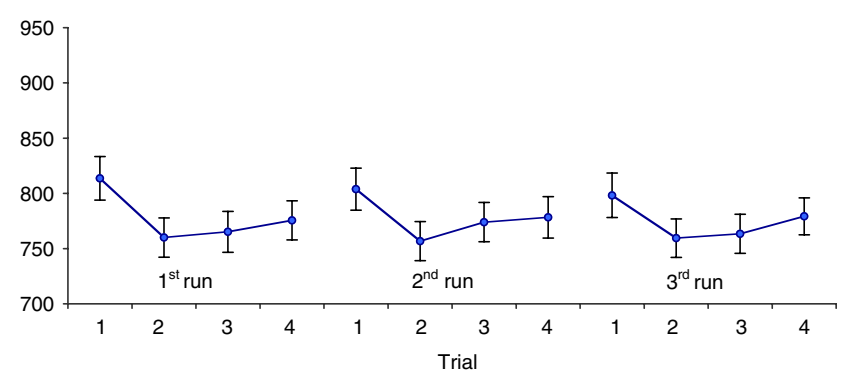

Fig. 3 Means of median response time (ms) and standard error in Experiment 2 as a function of trial number (1/2/3/4) over the three taskrun types (from left to right: first run, second run, and third run)

ment 1 , no significant reduction in restart cost across task repetition runs was observed.

A $3 \times 4$ (Run type $\times$ Trial) repeated measures ANOVA on error rates (see Table 2) revealed a significant main effect of run type, $F(2,38)=3.79, P<0.05$. On average, the participants made significantly more errors in first $(3.09 \%)$ than in second run $(2.32 \%)$, with $F(1,39)=7.59$, $P=0.009$. The main effect of trial $[F(3,37)=2.09$, $P=0.12]$ and the interaction $(F<1)$ were not significant. The task runs included in the analysis contained on average a low error rate of $2.59 \%$.

\section{Comparison between Experiments 1 and 2}

The influence of task predictability on task-repetition performance within a task-switching context was tested by comparing the repetition performance in Experiment 1 (unpredictable task order) with that in Experiment 2 (predictable task order). To do this, we conducted a $3 \times 2 \times 2$ ANOVA, with Run type (first run, second run, and third run), Trial (first and subsequent) and Experiment (1 and 2) on median RTs and errors. The two levels of Trial, namely, first and subsequent, referred to performance on first trials of a repetition run and the average performance on Trials 2, 3 , and 4. The ANOVA on RTs yielded a significant twoway interaction between trial and experiment $[F(1,98)=$ 9.69, $P<0.005]$. The restart cost, measured as the difference between first and subsequent trials, were significantly smaller in Experiment 2 than in Experiment 1 (47 vs. 85 $\mathrm{ms})$. Next to this reduction of restart cost, performance on subsequent task-repetition trials also generally improved in Experiment 2 relative to Experiment $1[F(1,98)=5.02$, $P=0.027$, for the main effect of Experiment on subsequent trials]. This improvement on subsequent trials in repetition runs in Experiment 2 indicated the presence of significant mixing cost in Experiment 1 relative to Experiment 2. The ANOVA on errors yielded no relevant main effects or interactions concerning the specific questions of the betweenexperiments comparison. 


\section{Discussion}

In the present experiment, we again replicated persistent restart cost. The comparison between Experiment 2 and 1 showed a general benefit of predictable task order for all task repetitions in Experiment 2 and thus indicate the existence of mixing cost in Experiment 1. This finding suggests that the cognitive system can use task predictability to prepare task repetitions (see also Koch, 2005, 2008; Monsell, et al., 2003).

Furthermore, Experiment 2 showed smaller restart cost compared to Experiment 1. This finding of a predictability benefit, specific for first trials after all task repetitions has at least two important theoretical implications. First, it suggests that cue-based task activation is receptive to task unpredictability when switching between tasks. Second, as restart cost decreased only to a certain amount and was not eliminated due to task predictability, our data also suggest that restart cost cannot be fully explained in terms of cuebased task activation needed to resolve unpredictable task order (see Allport \& Wylie, 2000, Experiment 3). The question that still remains is why the cognitive system needs to activate the relevant task even with predictable tasks. In Experiment 2, while tasks were predictable, switching between tasks still remained. It is, therefore, possible that a recent, previous activation of the currently incorrect task still induced some residual interference between the two competing tasks. Accordingly, the cuebased task activation could still be beneficial in resolving this interference. In Experiment 3, we tested this possible explanation.

\section{Experiment 3}

In Experiment 3, we tested the idea that the restart cost mainly reflects cue-based task activation needed to control the task interference that might arise from recent activation of the competing task. While in the previous two experiments, the activation of competing task might have generated task interference due to task switching, in the present experiment, we minimized the interference between the two tasks by introducing predictable task order in single-task conditions (i.e., no task switching involved). Task runs were induced in the same way here as in both preceding experiments, that is, by cue presentations. If restart cost is mainly generated by cuebased task activation needed to resolve task interference, then cue presentations in the present experiment should induce significantly smaller restart cost than in Experiment 2.

Furthermore, our data so far showed that task predictability was generally beneficial for all task repetitions.
Therefore, the second aim of this experiment was to examine the role of both task predictability and task interference in mixing cost. Mixing cost was measured here by comparing the performance in the task-repetition runs of Experiment 3, in which bivalent stimuli occurred under single-task conditions, with that of Experiment 2, in which bivalent stimuli occurred under mixed-tasks conditions. If mixing cost is mainly due to managing the interference that arises when switching between the competing tasks, then we expect to find mixing cost when comparing task repetitions in Experiment 3 (single-task conditions) to that in Experiment 2 (mixed-tasks conditions). If, however, mixing cost mainly arises due to task unpredictability that needs to be managed by cognitive system, then comparing task repetitions between Experiments 3 and 2 (both with predictable task order) should show no mixing cost.

\section{Method}

\section{Participants}

Thirty participants, 24 women and 6 men, were paid for taking part in this experiment. Their ages varied between 18 and 41 years, with a mean age of 22.2 years. Nine participants were left-handed, and the remaining 21 were right-handed. All participants had normal or corrected-tonormal vision.

\section{Stimuli and tasks}

The stimuli and tasks were as in Experiments 1 and 2.

\section{Procedure and design}

The procedure and the design of this experiment were identical to Experiment 1, except for one critical difference: Switching between the two tasks was excluded from this experiment. To achieve this, the experiment was divided in two parts. Each part started with 10 practice runs, followed by 82 experimental runs that were separated by one break in the middle. The two warming up runs in each experimental part were the first experimental run and the one just after the break in that part. In both parts, just one of the two tasks was required. In this way, this experiment included one transition point. One half of the participants started with the color task and finished with the shape task, while the other half of the participants followed the reversed sequence. Accordingly, the experiment contained (164) repetition runs only, except for the transition point in the middle of the experiment. As in Experiment 1, these runs consisted of a task cue followed by a run of four trials. 
Results

For RT analysis, the repeated measures ANOVA with Trial as the independent variable yielded no significant main effect (with 768, 759, 771, and $771 \mathrm{~ms}$ for Trial 1, 2, 3, and 4 , respectively). Even focusing the analysis on the experimental runs after the transition point introduced in the middle of the experiment revealed no significant restart cost. This was done to test the possibility of detecting restart cost when centering the analysis on the performance just after the only task-switch point in this experiment and after the 10 practice runs.

Submitting the error rates to the repeated measures ANOVA revealed a significant main effect of trial, $F(3,27)$ $=3.79, P<0.05$. The participants made fewer errors in Trial $1(1.73 \%)$ when compared to Trial $2[2.52 \% ; F(1,29)$ $=7.09, P=0.013]$ and Trial $4[2.52 \% ; F(1,29)=9.83$, $P=0.004]$ but not to Trial $3[2.35 \% ; F(1,29)=1.93$, $P=0.18]$. The task runs included in the analysis contained, on average a low error rate of $2.28 \%$.

\section{Comparison between Experiments 2 and 3}

The performance in task-repetition runs of Experiment 3 (single-task conditions) was compared with that of Experiment 2 (mixed-tasks conditions with predictable task order). To this end, $2 \times 2$ ANOVAs, with Trial (first and subsequent) and Experiment (2 and 3 ) were conducted on median RTs and errors. Also in this between-experiments comparison, the level "first" referred to the performance on first trials of a run and the level "subsequent" to the average performance on Trials 2, 3, and 4. The ANOVA on RTs yielded a significant interaction between trial and experiment, $F(1,68)=8.95, P<0.005$. Significantly larger restart cost was observed for Experiment 2 relative to Experiment 3 [47 vs. $9 \mathrm{~ms} ; F(1,39)=23.06, P<0.001$ ] Importantly, no difference in performance on subsequent trials, that is, no mixing cost was observed between the two experiments $(F<1)$. Finally, error rates yielded no relevant effects.

\section{Discussion}

The data of Experiment 3 showed no restart cost. Moreover, we observed even an improvement in performance on first trials in terms of error rates. These two findings indicate that restart cost arises only under mixed-tasks conditions. Activating the relevant task seems to be an effortful process reflected as performance cost on cued trials in situations when task interference arises due to recent activation of the competing task. If, however, task interference is reduced by introducing single-task conditions, like in Experiment 3, (re)activating the current task with each cue presentation becomes less crucial for task execution. It is even possible that cues were just visually encoded here, which induced no significant delay on first trials.

Importantly, no mixing cost was observed between Experiment 2 and Experiment 3. This finding suggests that mixing cost arises when task order is not predictable. Our data, therefore, suggest that mixing cost originates from limited preparation on repetition trials when it is not predictable which task will be required next.

\section{General discussion}

The present study investigated the cognitive mechanisms underlying restart cost and mixing cost in cued task switching. With unpredictable task order (Experiment 1), we observed restart cost that decreased to a certain amount when successively repeating the tasks. However, introducing predictable task order in task switching (i.e., mixed tasks) resulted not only in a smaller restart cost, but also in a generally improved task-repetition performance within a run. Accordingly, we found mixing cost on the non-cued repetition trials when task order was unpredictable (Experiment 1) compared to predictable task order (Experiment 2) within mixed-tasks conditions. Finally, Experiment 2 showed substantial restart cost compared to the single-task conditions in Experiment 3, but Experiment 2 showed no mixing cost relative to Experiment 3.

The persistent restart cost found with both unpredictable and predictable mixed tasks but not with single-task conditions, has several important theoretical implications. To start with, this finding suggests that restart cost develops only under situations of mixed tasks, that is, whenever task switching is involved. This is an important observation as it has implications for every model trying to account for taskswitching performance, since task repetitions function as a sort of base-line performance in task switching research. It is, therefore, critical to keep in mind the possible presence of restart cost even in the base-line measure when examining task switching. As to the mechanism behind the observed restart cost, our data imply processes involved in cue-based task activation needed to manage task interference as the main contributor to restart cost. It seems that a recent activation of the competing task generates the interference between the tasks that needs to be resolved by the cognitive system. Accordingly, our data show that reducing the task interference by introducing single task conditions induced no restart cost. These findings are in line with the idea proposed by Altmann (2002; see also Allport \& Wylie, 2000), who suggested that cognitive adaptations that enable the system to switch between the interfering tasks are, among others, reflected in restart cost. According to Altmann, during the encoding of the appropriate cue, the 
activation of the relevant task representation recovers allowing for the correct task execution. The effort that it takes to (re)build the episodic representation in memory is reflected in restart cost. The absence of restart cost observed for single tasks in this study provides additional evidence for Altmann's account, which implies that when task interference is significantly reduced (and no cognitive adaptations are needed), (re)activating the current task with each cue presentation becomes less crucial for task execution. As some other studies have shown that task interference can also be generated on the level of the specific S-R mappings (e.g., Waszak et al., 2003), it might also be interesting to test the involvement of stimulus priming in restart cost in future research.

Next to task interference as the main contributor of restart cost, our data show that restart cost is also affected by task predictability. Giving participants the opportunity to prepare the tasks based on sequential task predictability (Koch, 2005, 2008), the restart cost reduced. This preparatory reduction of the restart cost was also found by Poljac et al. (2006), who showed that providing ample time to prepare a task in advance reduces restart cost. In addition to this, the data of the present study argue against two alternative explanations for restart cost. First, one could assume that restart cost simply originates from any temporal break that disturbs the execution of the ongoing task. Separating runs of trials by cue presentations immediately implies a temporal separation of task execution (see Lien \& Ruthruff, 2004). If this were the case, cue presentations in Experiment 3 of the present study should have produced some performance impairment on cued trials and certainly not a performance improvement, which was observed in terms of errors. Accordingly, this alternative explanation seems highly unlikely. Second, Gopher et al. (2000) proposed that performing a run of trials belonging to the same task stimulates the development of a kind of task rhythm. Every time this rhythm is interrupted, the system needs some extra time to generate the first response of the following run of trials. According to this view, disturbing the rhythm of a sequential task execution might generate restart cost. However, again one would have expected to observe restart cost in single-task conditions, but the absence of restart cost in the present Experiment 3 offers no support for this view either.

Importantly, the present data suggest that restart cost and mixing cost are based on dissociable mechanisms. We found that predictable task order was generally beneficial for all task repetitions (see also e.g., Koch, 2005, 2008; Monsell et al., 2003). When task order was unpredictable, we observed mixing cost (i.e., as assessed by comparing the performance on the non-cued trials between Experiment 1, in which the task order was unpredictable, with that of Experiment 2, in which the task order was predictable). As task execution on the non-cued trials of task-repetition runs did not additionally improve by minimizing the task interference between the competing tasks (Experiment 3), our data imply that the optimal task execution on repetition trials depends on how predictable the identity of the approaching task is. Recently, Koch and Philipp (2005) observed that performance improvements on repetition trials due to adequate advance preparation (i.e., cuing interval) depended on task predictability in a similar way as mixing cost in the present study. Specifically, providing ample preparation time on task-repetition trials was beneficial for task performance only with unpredictable task sequences. If, however, tasks were predefined, providing more time for advance preparation of tasks had no effects on task execution. It thus seems that stimulus- and/or response-related processes on repetition trials profit from preparation based on sequential task predictability in such a way that the irrelevant stimulus feature no longer significantly interferes with the execution of the required task (see also Koch \& Allport, 2006). Hence, the data suggest that restart cost is due to cue-based task activation under mixedtasks conditions generally, whereas mixing cost arise specifically when task repetitions are unpredictable und thus relatively less prepared than in predictable tasks.

It is interesting to speculate though whether our results might also be related to the process of forming higher-order representations of task sequences and hierarchical control structures (e.g., Koch, Philipp, \& Gade, 2006; Lien \& Ruthruff, 2004; Luria \& Meiran, 2003; Schneider \& Logan, 2006). Since the tasks of the present study were organized in runs of four trials, task sequencing might have contributed to the findings of our study. Along these lines, one could argue that the observed restart cost is due to (re)activating of a specific task "chunk" (e.g., Koch et al., 2006). Accordingly, within-chunk facilitation might have contributed to the performance benefit observed with predictable task order on both cued and non-cued trials. Importantly, while this speculation is certainly interesting as a potential alternative account for restart cost, it does not seem to offer a viable explanation for mixing cost. Nevertheless, in future work it clearly seems desirable to better relate the empirical phenomena of restart cost and mixing cost to higher-order processes, such as task chunking.

In conclusion, the data of the present study suggest that restart cost and mixing cost are based on dissociable mechanisms. For restart cost, this study implies that this cost arises from processes involved in cue-based task activation that is needed to resolve task interference generated by the activation of the competing task whenever switching between tasks is required. For mixing cost, the data imply that the optimal task execution on repetition trials is dependent on task predictability. Therefore, we suggest that mixing cost arise from limited preparation on repetition trials in situations of unpredictable task order. 
Acknowledgments We thank Dennis Pasveer for programming the experiments in E-Prime, Gerard van Oijen for technical support, and Lilla Magyari for collecting the data. Also, we would like to thank Andrea M. Philipp for brainstorming sessions, in which the ideas for this paper were elaborated. We thank Erik Altmann and Thomas Kleinsorge for valuable and constructive comments on an earlier version of this article.

Open Access This article is distributed under the terms of the Creative Commons Attribution Noncommercial License which permits any noncommercial use, distribution, and reproduction in any medium, provided the original author(s) and source are credited.

\section{References}

Allport, A., Styles, E. A., \& Hsieh, S. (1994). Shifting intentional set: Exploring the dynamic control of tasks. In C. Umilta, \& M. Moscovitch (Eds.), Attention and performance XV (pp. 421-452). Cambridge: MIT Press.

Allport, A., \& Wylie, G. (2000). Task switching, stimulus-response bindings, and negative priming. In S. Monsell, \& J. Driver (Eds.), Control of cognitive processes: Attention and performance XVIII (pp. 357-376). Cambridge: MIT Press.

Altmann, E.M. (2002). Functional decay of memory for tasks. Psychological Research, 66, 287-297.

Altmann, E.M. (2004). Advanced preparation in task switching: What work is being done? Psychological Science, 15, 616-622.

Altmann, E.M. (2007). Comparing switch costs: Alternating runs and explicit cuing. Journal of Experimental Psychology: Learning, Memory, and Cognition, 33, 475-483.

Altmann, E.M., \& Gray, W.D. (2002). Forgetting to remember: The functional relationship of decay and interference. Psychological Science, 13, 27-33.

Bishop, Y.M.M., Fienberg, S.E., \& Holland, P.W. (1975). Discrete multivariate analysis: Theory and practice. Cambridge: MIT Press.

Braver, T.S., Reynolds, J.R., \& Donaldson, D.I. (2003). Neural mechanisms of transient and sustained cognitive control during task switching. Neuron, 39, 713-726.

Gopher, D., Armony, L., \& Greenshpan, Y. (2000). Switching tasks and attention policies. Journal of Experimental Psychology: General, 129, 308-339.

Koch, I. (2005). Sequential task predictability in task switching. Psychonomic Bulletin \& Review. 12, 107-112.

Koch, I. (2008). Instruction effects in task switching. Psychonomic Bulletin \& Review (in press).

Koch, I., \& Allport, A. (2006). Cue-based preparation and stimulusbased priming of tasks in task switching. Memory and Cognition, $34,433-444$.
Koch, I., \& Philipp, A.M. (2005). Effects of response selection on the task repetition benefit in task switching. Memory and Cognition, 33, 624-634.

Koch, I., Philipp, A.M., \& Gade, M. (2006). Chunking in task sequences modulates task inhibition. Psychological Science, 17, 346-350.

Koch, I., Prinz, W., \& Allport, A. (2005). Involuntary retrieval in alphabet-arithmetic tasks: Task-mixing and task switching costs. Psychological Research, 69, 252-261.

Lien, M.-C., \& Ruthruff, E. (2004). Task switching in a hierarchical task structure: Evidence for the fragility of the task repetition benefit. Journal of Experimental Psychology: Learning, Memory and Cognition, 30, 697-713.

Los, S.A. (1996). On the origin of mixing costs: Exploring information processing in pure and mixed blocks of trials. Acta Psychologica, 94, 145-188.

Luria, R., \& Meiran, N. (2003). Online order control in the psychological refractory period paradigm. Journal of Experimental Psychology: Human Perception and Performance, 29, 556-574.

Meiran, N., Chorev, Z., \& Sapir, A. (2000). Component processes in task switching. Cognitive Psychology, 41, 211-253.

Milan, E.G., Sanabria, D., Tornay, F.J., \& Gonzalez, A. (2005). Exploring task-set reconfiguration with random task sequences. Acta Psychologica, 118, 319-331.

Monsell, S. (2003). Task Switching. Trends in Cognitive Sciences, 7, 134-140.

Monsell, S., Sumner, P., \& Waters, H. (2003). Task-set reconfiguration with predictable and unpredictable task switches. Memory \& Cognition, 31, 327-342.

Poljac, E., De Haan, A., \& Van Galen, G.P. (2006). Current task activation predicts general effects of advanced preparation in task switching. Experimental Psychology, 53, 260-267.

Rogers, R.D., \& Monsell, S. (1995). Costs of a predictable switch between simple cognitive tasks. Journal of Experimental Psychology: General, 124, 207-231.

Rubin, O., \& Koch, I. (2006). Exogenous influences on task set activation in task switching. The Quarterly Journal of Experimental Psychology, 59A, 1033-1046.

Rubin, O., \& Meiran, N. (2005). On the origins of the task mixing cost in the cuing task-switching paradigm. Experimental Psychology: Learning, Memory, and Cognition, 31, 1477-1491.

Schneider, D.W., \& Logan, G.D. (2006). Hierarchical control of cognitive processes: Switching tasks in sequences. Journal of Experimental Psychology: General, 135, 623-640.

Tornay, F.J., \& Milan, E.G. (2001). A more complete task-set reconfiguration in random than in predictable task switch. Quarterly Journal of Experimental Psychology, 54A, 785-803.

Waszak, F., Hommel, B., \& Allport, A. (2003). Task-switching and long-term priming: Role of episodic stimulus-task bindings in task-shift costs. Cognitive Psychology, 46, 361-413. 